\title{
SCATTERING THEORY
}

\author{
BY PETER D. LAX ${ }^{1}$ AND RALPH S. PHILLIPS ${ }^{2}$ \\ Communicated September 6, 1963
}

1. Let $H$ be a Hilbert space, $U(t)$ a group of unitary operators. A closed subspace $D_{+}$of $H$ will be called outgoing if it has the following properties:

(i) $U(t) D_{+} \subset D_{+}$for $t$ positive.

(ii) $\cap_{t>0} U(t) D_{+}=\{0\}$.

(iii) $U_{t<0} U(t) D_{+}$dense in $H$.

A prototype of the above situation is when $H$ is $L_{2}(-\infty, \infty ; N)$, i.e., the space of square integrable functions on the whole real axis whose values lie in some accessory Hilbert space $N, U(t)$ is translation by $t$, and $D_{+}$is $L_{2}(0, \infty ; N)$.

THEOREM $1 .^{3}$ If $D_{+}$is outgoing for the group $U(t)$, then $H$ can be represented isometrically as $L_{2}(-\infty, \infty ; N)$ so that $U(t)$ is translation and $D_{+}$is the space of functions with support on the positive reals. This representation is unique up to isomorphisms of $N$.

We shall call this representation an outgoing translation representation of the group.

Taking the Fourier transform we obtain an outgoing spectral representation of the group $U(t)$, where elements of $D_{+}$are represented as functions in $A_{+}(N)$, that is the Fourier transform of $L(0, \infty ; N)$. According to the Paley-Wiener theorem $A_{+}(N)$ consists of boundary values of functions with values in $N$, analytic in the upper half-plane whose square integrals along lines $\operatorname{Im} z=$ const are uniformly bounded.

An incoming subspace $D_{-}$is defined similarly and an analogous

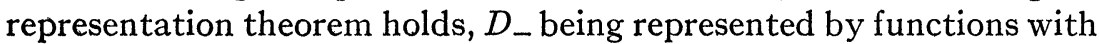
support on the negative axis, that is, by $L_{2}\left(-\infty, 0 ; N_{-}\right) . N_{-}$and $N$ are unitarily equivalent and will henceforth be identified. In the application to the wave equation there is a natural identification of $N$ and $N_{-}$.

Let $D_{+}$and $D_{-}$be outgoing and incoming subspaces respectively for the same unitary group, and suppose that $D_{+}$and $D_{-}$are orthogonal. To each function $f \in H$ there are associated two functions $k_{-}$ and $k_{+}$, the respective incoming and outgoing translation representa-

\footnotetext{
1 Sloan Fellow.

2 Sponsored by the National Science Foundation, contract NSF-G 16434.

3 We were informed by Professor Sinai that he has obtained and used a similar theorem.
} 
tions of $f$. The mapping $k_{-} \rightarrow k_{+}$, denoted by $S$, is called the scattering operator and has the following properties:

(i) $S$ is unitary.

(ii) $S$ commutes with translation.

(iii) $S$ maps $L_{2}(-\infty, 0, N)$ into $L_{2}(-\infty, 0, N)$.

Properties (i) and (ii) follow from the fact that $S$ is defined in terms of two different unitary translation representations of the same group. To deduce property (iii), we note that every function in $L_{2}(-\infty, 0, N)$ of the incoming representation corresponds to an element $f$ of $D_{-}$; since we have assumed that $D_{-}$is orthogonal to $D_{+}$it follows that the function representing $f$ in the outgoing representation will be orthogonal to $L_{2}(0, \infty, N)$, i.e., will belong to $L_{2}(-\infty, 0, N)$, as asserted in (iii).

We take now Fourier transforms and define the operator $\delta$ as $F S F^{-1}, F$ denoting the Fourier transformation. Properties (i)-(iii) for $S$ translate into

(i) $\delta$ is unitary.

(ii) ${ }^{\prime} S$ commutes with multiplication by scalar functions.

(iii)' $\&$ maps $A(N)$ into $A(N)$

where $A(N)$ is the Fourier transform of $L_{2}(-\infty, 0, N)$ and thus consists of the boundary values of functions analytic in the lower halfplane.

According to a simple special case of a theorem of Segal and Fourès [13], an operator with properties (i)', (ii)' and (iii)' is multiplication by an operator valued function $\delta(z)$, mapping $N$ into $N$, with the following properties:

THEOREM 2. (a) $S(z)$ is analytic in the lower half-plane.

(b) The norm of $\delta(z)$ is not greater than one for every $z$.

(c) $\mathcal{S}(z)$ is unitary for $z$ real.

$S(z)$ is the Heisenberg scattering matrix. Extending the terminology of Beurling [1], to the operator case $\delta(z)$ is also an inner factor. ${ }^{4}$

2. Let $D_{+}$and $D_{-}$be as before and denote by $P_{+}$and $P_{-}$orthogonal projection onto the orthogonal complements of $D_{+}$and $D_{-}$respectively. Consider the one-parameter family of operators $Z(t)$ defined as

$$
Z(t)=P_{+} U(t) P_{-} .
$$

It follows easily that for positive $t, Z(t)$ annihilates both $D_{+}$and $D_{-}$; consider $Z(t)$ for positive values of $t$ and acting on $K=H \ominus D_{+} \ominus D_{-}$.

THEOREM 3. $Z(t)$ forms a semigroup over $K$.

\footnotetext{
${ }^{4}$ See [1], [7] and [2] for the theory of inner factors.
} 
This is very easy to prove directly from the postulated relations of $D_{+}$and $D_{-}$to each other and $U(t)$. It also follows from the interpretation of $Z(t)$ in the, say, outgoing translation representation. For, since $H \ominus D_{+}$is represented by $L_{2}(0, \infty, N)$ and $D_{-}$by $L_{2}(0, \infty, N), K$ is represented by

$$
K \Leftrightarrow L_{2}(0, \infty, N) \ominus L_{2}(0, \infty, N),
$$

and the action of $Z(t)$ consists in shifting to the right followed by restriction to the negative real axis.

A subspace of functions $K$ which is mapped into itself under such an operation is called a translation invariant space. It is not surprising that $K$ and $Z(t)$ can be so represented, since according to a simple generalization of a theorem of Beurling every contraction semigroup $Z(t)$ can be so represented, provided that for every $u$ in $K,\|Z(t) u\|$ tends to zero as $t$ tends to infinity.

In the outgoing spectral representation $K$ is represented as

$$
A(N) \ominus S A(N) ;
$$

$\delta$ is called the inner factor associated with the translation invariant space $K$. Again this is no surprise since according to a generalization due to Lax, [6], [7] of a theorem of Beurling, see also Halmos [2], the orthogonal complement of the Fourier transform of every translation invariant space is of the form $\delta A$, where $\delta$ is an inner factor. The importance of this representation is that the associated inner factor contains almost complete information about the spectrum of $Z(t)$ over $K$ :

TheORem $4 .{ }^{5}$ (a) Let $\mu$ be a complex number with negative real part; $\mu$ belongs to the resolvent set of the infinitesimal generator $B$ of $Z(t)$ if and only if the operator

$$
\delta(i \bar{\mu})
$$

is invertible.

(b) Let $\lambda$ be a complex number of absolute value less than one; $\lambda$ belongs to the resolvent set of $Z(t)$ if and only if

$$
\S\left(\frac{i}{t} \bar{\mu}\right)
$$

is invertible for all numbers $\mu$ for which $e^{\mu t}=\lambda$, and if the norms of the inverses are uniformly bounded for all such $\mu$.

As corollary we obtain another proof of the well-known result of Phillips, see [11] or [3], that if $\mu$ belongs to the spectrum of $B$ then

' See [10] for the scalar case. 
$e^{\mu t}$ belongs to the spectrum of $Z(t)$, but in general not conversely.

THEOREM 5. If for some value of $T,\|Z(T)\|=a<1$, then $\$(z)$ can be continued analytically into the strip $\operatorname{Im} z \leqq-\log a / T$.

Analogous expressions can be derived for the location of the spectrum of any function of $Z(t)$ and $B$; from this we deduce

TheOREM $5^{\prime}$. If for some $T$ and some $\mu, Z(T)(B-\mu)^{-1}$ is completely continuous, then $S(z)$ can be continued into the upper half-plane as a meromorphic function.

The definition of the scattering matrix depends on the choice of a pair of orthogonal incoming and outgoing subspaces. Let us call two outgoing subspaces $D_{+}$and $D_{+}^{\prime}$ equivalent if for sufficiently large positive $T, U(-T) D_{+}$contains $D_{+}^{\prime}$ and $U(T) D_{+}$is contained in $D_{+}^{\prime}$. Following the derivation of Theorem 2 one can easily show that the outgoing spectral representations with respect to $D_{+}$and $D_{+}^{\prime}$ are related by multiplication by an operator valued function $\Re(z)$ which is entire analytic, of exponential growth, and unitary on the real axis. Such a function satisfies the relation

$$
\mathfrak{T}^{*}(\bar{z})=\mathfrak{T C}^{-1}(z)
$$

for $z$ real, so by analytic continuation for all $z$; this shows that $\mathfrak{T}^{-1}$ exists for all $z$ and is of exponential growth.

Suppose that $D_{+}, D_{-}$and $D_{+}^{\prime}, D_{-}^{\prime}$ are two pairs of orthogonal incoming and outgoing subspaces which are equivalent. Then the associated scattering matrices are related by

$$
\mathfrak{S}^{\prime}=\mathfrak{M C}_{+} \mathrm{STI}_{-}^{-1} \text {. }
$$

Since the factors $\mathfrak{N C}_{+}, \mathfrak{N}_{-}$and their inverses are uniformly bounded in any strip, we conclude from Theorem 4 that the associated semigroups have the same spectrum.

Choose in particular $D_{+}^{a}$ as $U(a) D_{+}$, and $D_{-}^{a}$ as $U(-a) D_{-}, a$ positive. As is easily shown,

$$
\mathcal{S}^{a}(z)=e^{2 i a z} \mathcal{S}(z) .
$$

We denote the operator $Z(t)$ corresponding to $D_{ \pm}^{a}$ by $Z_{a}(t)$.

THEOREM 6. If $f^{b}$ is an eigenvector of $Z_{b}(t)$ with eigenvalue $e^{\mu t}$, then for $a<b$

$$
f^{a}=P_{+}^{a} f^{b}
$$

is an eigenvector of $Z_{a}(t)$ with the same eigenvalue. 
3. Let $H_{0}$ denote the Hilbert space of pairs of functions $f=\left[f_{1}, f_{2}\right]$ defined in $R_{n}$, normed by the energy norm:

$$
\|f\|^{2}=\int\left(\left|D f_{1}\right|^{2}+\left|f_{2}\right|^{2}\right) d x .
$$

Define $U_{0}(t)$ as the operator which relates the Cauchy data at time zero of solutions of the wave equation to their Cauchy data at time $t$. $U_{0}(t)$ forms a one-parameter group of unitary operators mapping $H_{0}$ onto $H_{0}$ (conservation of energy).

Consider a smooth, bounded, reflecting obstacle. Denote by $H$ the subspace of $H_{0}$ consisting of pairs of functions which vanish inside the obstacle, and denote by $U(t)$ the operator which relates the initial data to data at time $t$ of solutions of the wave equation defined outside of the obstacle and vanishing on it. $U(t)$ forms a one-parameter group of unitary operators mapping $H$ onto $H$.

We shall call a solution of the wave equation defined for all values of $x$ and $t$ outgoing (incoming) if it vanishes inside the cone $|x|$ $<t(|x|<-t)$. We denote by $D_{ \pm}^{0}$ the data at time zero of outgoing (incoming) solutions.

$D_{+}^{0}$ and $D_{-}^{0}$ are outgoing and incoming subspaces for the group $U_{0}(t)$ in the sense of $\$ 1$; the first two properties are obviously satisfied and the third is an easy consequence of Huygens' principle. As shown in [9], $D_{+}^{0}$ and $D_{-}^{0}$ are orthogonal for $n$ odd; we give here a new proof based on an explicit form for the translation representation.

We start with the representation of functions in terms of their Radon transforms:

$$
f(x)=\int_{|\omega|=1} h(x \cdot \omega, \omega) d \omega
$$

where $h(s, \omega)$, the Radon transform of $f$, is a function of $s$ and $\omega$ defined for all real $s$ and all vectors $\omega$ on $S_{n-1}$ which is even:

$$
h(-s,-\omega)=h(s, \omega) .
$$

A Parseval relation holds:

$$
\|f\|^{2}=\|h\|_{-(n-1) / 2}
$$

where we define

$$
\|h\|_{-q}^{2}=\int|k(s, \omega)|^{2} d s d \omega, \quad \frac{\partial^{q}}{\partial s^{q}} k=h .
$$


Corollary.

$$
\|f\|_{1}=\|h\|_{-(n-3) / 2}
$$

Let $h_{1}$ and $h_{2}$ denote the Radon transforms of $f_{1}$ and $f_{2}$ respectively and define $h$ as

$$
h=h_{1}-\int h_{2}
$$

It can be verified immediately that the function

$$
u(x, t)=\int h(x \cdot \omega-t, \omega) d \omega
$$

is a solution of the wave equation and that its initial data are $f_{1}$ and $f_{2}$. Furthermore, by (3.2) and (3.2)',

$$
\|f\|=\|h\|_{-(n-3) / 2}
$$

Let $k$ be the $(n-3) / 2$ fold integral of $h$; regarding $k$ as a function of $s$ whose values lie in the Hilbert space $N=L_{2}\left(S_{n-1}\right)$, we conclude from (3.3), (3.4) that $f \rightarrow k$ is a translation representation for $U_{0}(t)$. We claim that for $n$ odd this representation is both incoming and outgoing.

It follows from (3.3) that if $h(s)$ vanishes for negative (positive) values of $s$, then $u(x, t)$ vanishes in the forward (backward) cone $|x|<t(|x|<-t)$. Conversely:

THEOREM 7. If $u(x, t)$ vanishes in the forward (backward) cone then $h$ vanishes on the negative (positive) axis.

SkETCH OF PROOF. If $u$ vanishes in the forward cone, all its space derivatives vanish on the positive $t$ axis:

$$
0=\left(D_{x}^{j} u\right)(0, t)=\int \omega^{j} h^{|j|}(-t, \omega) d \omega .
$$

Multiply this by any smooth test function $\phi(t)$ whose support lies on the positive $t$-axis, integrate with respect to $t$ and perform $|j|$ integrations by parts:

$$
0=\int \omega^{j} h(-t, \omega) \phi^{|j|}(t) d \omega d t
$$

From (3.5) and the fact that $h$ has finite $(3-n) / 2$ norm it follows by an approximation procedure that for every smooth test function $\chi(t)$ with compact support on the positive $t$ axis and every multi index $j$ 


$$
\iint h(-t, \omega) \omega^{i} \chi(t) d \omega d t=0
$$

But this implies that $h(s)$ vanishes for $s$ negative; then so does $k$ for $n$ odd.

REMARK. In the proof we only used the fact that $u(x, t)$ has a zero of infinite order on the positive $t$-axis; thus we have shown that this condition implies that $u$ vanishes in the forward cone-a new proof for a special case of a theorem of Fritz John.

Corollary. For $n$ odd, $D_{+}$and $D_{-}$are orthogonal.

For the group $U_{0}(t)$ we have found a representation with $D_{+}^{0}$ and $D^{0}$ - as outgoing and incoming subspaces. The associated scattering operator is the identity. We turn now to the group $U(t)$ and take for $D_{+}$and $D_{-}$the initial data of solutions which vanish in $|x|<\rho+t$ for $t>0$ and $|x|<\rho-t$ for $t<0$ respectively; ${ }^{6}$ we claim that these subspaces are outgoing and incoming respectively for $U(t)$ : Properties (i) and (ii) are immediate while property (iii) is proved in [8]. $D_{+}$ and $D_{-}$are orthogonal since they are subspaces of $D_{+}^{0}$ and $D_{-}^{0}$. Thus there exists an associated scattering matrix. Conversely, we can prove

THEOREM 8. The scattering matrix uniquely determines the scattering obstacle.

In [9], Cathleen Morawetz and the authors have shown that for star-shaped obstacles $\|Z(t)\|$ is less than one for $t$ large enough. By Theorem 5 it follows that the associated scattering matrix can be continued analytically into a strip $0 \leqq \operatorname{Im} z \leqq \tau$. For any obstacle we have this result:

THEOREM 9. For $\operatorname{Re} \lambda$ positive $Z(2 \rho)(B-\lambda)^{-1}$ is completely continuous.

By Theorem $5^{\prime}$ this implies that $S(z)$ can be continued into the upper half-plane as a meromorphic function. This implies that the zeros of $\delta(z)$ in the lower half-plane are discrete; furthermore, for each $z, \S(z)$ has a closed range whose codimension is finite and equal to the dimension of the nullspace of $s(z)$.

SKETCH OF PROOF.

Lemma 1. The operator

$$
M=U(2 \rho)-U_{0}(2 \rho)
$$

\footnotetext{
${ }^{\circ} \rho$ is chosen so large that the sphere $|x|<\rho$ contains the obstacle.
} 
annihilates all $f$ in $H$ which vanish in $|x|<3 \rho$.

LEMma 2. $U_{0}(2 \rho)$ maps the complement of $D_{-}$into $D_{+}$.

Lemma 2 implies that $P_{+} U_{0}(2 \rho) P_{-}=0$, whence for $t \geqq 2 \rho$

$$
Z(t)=P_{+} U(t) P_{-}=P_{+} M U(t-2 \rho) P_{-} .
$$

So

$$
Z(2 \rho)(B-\lambda)^{-1}=e^{2 \lambda \rho} \int_{2 \rho}^{\infty} Z(t) e^{-\lambda t} d t
$$

$$
=e^{2 \lambda \rho} P_{+} M \int_{2 \rho}^{\infty} U(t-2 \rho) e^{-\lambda t} d t P_{-}=P_{+} M(A-\lambda)^{-1} P_{-},
$$

where $A$ denotes the infinitesimal generator of $U(t)$. It is easy to show that $(A-\lambda)^{-1}$ raises by one the degree of differentiability; since by Lemma 1 the value of $M f$ does not depend on the values of $f$ outside the sphere $|x| \leqq 3 \rho$, it follows by Rellich's compactness criterion that $M(A-\lambda)^{-1}$ is a completely continuous operator.

For the pure initial value problem for hyperbolic equations with variable coefficients it is known that the sharp propagation of signals is along characteristic rays. This generalized Huygens principle can be reformulated as follows:

Let $G_{1}$ and $G_{2}$ be two closed sets in $R_{n}$ with the property that no characteristic ray starting at time zero in $G_{1}$ passes through $G_{2}$ at time $t$. Let $P_{1}$ and $P_{2}$ be operators such that the range of $P_{1}$ consists of functions which vanish outside of $G_{1}$, while $P_{2}$ annihilates all functions whose support lies outside $G_{2}$. Then

$$
P_{2} U_{0}(t) P_{1},
$$

is completely continuous.

We believe that this principle also holds for the mixed problem as well (for general hyperbolic equations with variable coefficients), provided that rays are interpreted as reflected rays. A demonstration of this for the interior problem for convex domains has been given by Povsner and Suharevskil [12].

We say that an obstacle has property $L$ if there exists a number $l$ such that any ray starting in the sphere $|x| \leqq 3 \rho$ leaves the sphere $|x| \leqq 3 \rho$ after time $l$.

REMARK. Star-shaped obstacles have property $L$.

Assuming the generalized Huygens principle to hold we assert:

THEOREM 10. $Z(t)$ is eventually compact if and only if the obstacle has property $L$. 
Proof. The identity (see [9])

$$
Z(t+4 \rho)=P_{+} M U(t) M P_{-}
$$

follows similarly as (3.6). Take both $G_{1}$ and $G_{2}$ as the sphere $|x| \leqq 3 \rho$; the operator $M P_{\text {- }}$ has the property required of $P_{1}$ while $M$ has the property required of $P_{2}$. So by the generalized Huygens principle $Z(l+4 \rho)$ is completely continuous.

The necessity of property $L$ follows from known properties of propagation of high frequency signals along rays.

Theorem 10 implies that $Z(t)$ has a standard discrete spectrum. There can be no eigenvalue of absolute value one since this would correspond to a solution of the wave equation which is a purely imaginary exponential in time, and according to a theorem of Rellich there are no such solutions with finite energy. Thus the spectral radius of $Z(t)$ is less than one; by the Gelfand formula we conclude that $\|Z(t)\|$ decays exponentially. Thus Theorem 10 gives another proof of the result of [9].

Similar reasoning gives the following result: let $f$ be any element of $K, \sum a_{j} f_{j}$ its formal Fourier expansion in terms of the eigenfunctions of $Z(t)$; then

$$
\sum a_{j} e^{\mu_{j} t} f_{j}
$$

is an asymptotic expansion for $Z(t) f$.

Next we wish to characterize the eigenvalues and eigenfunctions of the generator $B$ of $Z(t)$. For this purpose we say that a solution of the reduced wave equation

$$
\Delta u-\mu^{2} u=0
$$

in the exterior domain is outgoing if the free space solution of the wave equation with initial data $f=[u,-\mu u]$, in symbols $U_{0}(t) f$, vanishes for $|x|<t-\rho$ for all $t>\rho$. This notion is equivalent with the Sommerfeld definition of outgoing when $\mu$ is imaginary. Moreover for arbitrary $\mu$ in the case $n=3$ such a solution of the reduced wave equation can be represented as

$$
u(x)=\frac{1}{4 \pi} \int_{\mathrm{r}}\left(u \frac{\partial u}{\partial n}-v \frac{\partial u}{\partial n}\right) d S_{y}
$$

where $v=e^{\mu r} / r, r=|x-y|$, and $\Gamma$ is any smooth surface containing the obstacle but not containing $x$. The converse is also true.

THEOREM 11. $\mu$ is an eigenvalue of the generator of $Z(t)$ if and only if there exists an outgoing solution of the reduced wave equation (3.8) satisfying the boundary conditions. 
Sketch OF PROOF. We consider $Z_{a}(t)=P_{+}^{a} U(t) P_{-}^{a}$ as $a \rightarrow \infty$; in the limit this is simply $U(t)$. According to Theorem 4 the eigenvalues $\mu$ of the generator of $Z_{a}(t)$ are simply related to the zeros of the scattering operator; thus they are independent of $a \geqq \rho$. The eigenfunctions depend upon " $a$ " but according to Theorem 6 in a rather trivial fashion. In fact for $b>a \geqq \rho$,

$$
f^{a}=P_{+}^{a} f^{b}
$$

Since $P_{+}^{a}$ does not alter the data inside the sphere $|x|<a$, it follows that $f^{a}(x)=f^{b}(x)$ for $|x|<a$. This shows that the limit

$$
\lim _{a \rightarrow \infty} f^{a}(x) \equiv f(x)
$$

exists.

Each $f_{a}$ satisfies

$$
Z_{a}(t) f_{a}=e^{-\mu t} f_{a} .
$$

Since $Z_{a}(t) f=U(t) f$ for $|x|<a, f_{a}$ is a solution of the reduced wave equation there. So for $|x|<a, f_{a}$ is of the form

$$
f_{a}=\left(u_{a},-\mu u_{a}\right)
$$

$u_{a}$ a solution of the reduced wave equation

$$
\Delta u_{a}-\mu^{2} u_{a}=0
$$

which is zero on the obstacle. Since $u_{a}(x)=u_{b}(x)$ for $|x|<a$, the limit

$$
\lim _{a \rightarrow \infty} u_{a}=u
$$

exists. $u$ is in the exterior a solution of the reduced wave equation and is zero on the obstacle. The data $f=[u, \mu u]$ can be thought of as a generalized eigenfunction of $U(t)$; not only does it not lie in $H$, but it blows up exponentially in $|x| \cdot f^{\rho}$ is orthogonal to $D_{-}$and so is $f^{a}$ for all $a \geqq \rho$ by (3.9). As a consequence the free space solution $U_{0}(t) f$ vanishes in $|x|<t-\rho$ for $t>\rho$ so that $u$ is outgoing.

Conversely if $u$ is an outgoing solution of the reduced wave equation (3.8) satisfying the boundary conditions, then $e^{-\mu t}$ is an eigenvalue of $Z(t)$. To prove this one shows that the free space translation representation of $f=[u,-\mu u]$ is of the form

$$
h(s, \omega)= \begin{cases}0, & s<-\rho, \\ n(\omega) e^{\mu s}, & s>\rho .\end{cases}
$$

Setting 


$$
h_{a}(s, \omega)= \begin{cases}h(s, \omega), & s<a, \\ 0, & s>a,\end{cases}
$$

one proves that $h_{a}$ is the free space translation representation of the eigenfunction of $Z_{a}(t)$ corresponding to the eigenvalue $e^{-\mu t}$.

The above ideas can also be employed to obtain an explicit description of the incoming and outgoing spectral representations of $U(t)$ from which we will in turn be able to obtain an explicit formula for the scattering operator $\boldsymbol{s}(z)$. We shall denote by $\hat{f}_{0}, \hat{f}_{-}$, and $\hat{f}_{+}$ the free space, incoming, and outgoing spectral representations respectively of a given initial data $f$.

These spectral representations are given by scalar products of $f$ with certain improper eigenfunctions of $U_{0}(t)$, respectively $U(t)$. We shall show that these improper eigenfunctions consist of exponentials plus certain incoming and outgoing solutions. We recall that the free space spectral representation for $U_{0}(t)$ is simultaneously incoming and outgoing. Thus $D_{-}^{0}$ and $D_{+}^{0}$ map onto $A_{-}(N)$ and $A_{+}(N)$ respectively, while $D_{-}$and $D_{+}$map onto $e^{-i \rho z} A_{-}(N)$ and $e^{i \rho z} A_{+}(N)$ respectively. We shall limit our considerations to the case $n=3$.

ThEOREM 12 (SPECTRAL REPRESENTATION FOR $U_{0}(t)$ ).

$$
\hat{f}_{0}(z, \omega)=\left(f, \phi_{0}(\cdot, z, \omega)\right)
$$

where $\left(\right.$, ) denotes the $H_{0}$ inner product and

$$
4 \pi^{3 / 2} \phi_{0}(x, z, \omega)=\left[e^{-i z x \cdot \omega}, i z e^{-i z x \cdot \omega}\right] .
$$

The main tool employed in the derivation of (3.10) is the Fourier transform.

TheOREM 13 (INCOMING AND OUTGOING SPECTRAL REPRESENTATIONS FOR $U(t))$. Let $v_{+}\left(v_{-}\right)$be the outgoing (incoming) solution of the reduced wave equation

$$
\Delta v+z^{2} v=0
$$

satisfying $v+e^{-i z x \cdot \omega}=0$ on the obstacle. Set

$$
4 \pi^{3 / 2} \psi_{ \pm}(x, z, \omega)=\left[v_{ \pm}(x, z, \omega), i z v_{ \pm}(x, z, \omega)\right]
$$

and define

$$
\phi_{ \pm}=\phi_{0}+\psi_{ \pm}
$$

Then

$$
\hat{f}_{ \pm}(z, \omega)=\left(f, \phi_{\mp}(\cdot, z, \omega)\right),
$$

where the $($,$) denotes the inner product in H$. Note the switch in signs. 
SKETCH OF PROOF FOR THE INCOMING REPRESENTATION FORMULA. Step one. To verify (3.11) for data in $D_{-}$. It is required that data in $D_{\text {- }}$ have the same representation as in the free space spectral representation. This in turn requires that $\left(f, \psi_{+}\right)=0$ for all $f$ in $D_{-}$. Now for $f=U_{0}(-\tau-\rho) w$ where $w$ has support in $|x|<\tau$, it is clear that

$$
\left(f, \psi_{+}\right)=\left(w, U_{0}(\tau+\rho) \psi_{+}\right)=0
$$

since $U_{0}(\tau+\rho) \psi_{+}$vanishes for $|x|<\tau$. It is proved in [9] that linear combinations of such $f$ are dense in $D_{-}$and hence (3.11) shares with (3.10) the property of being an isometry in $D_{-}$.

Step two. Extend the isometric property of the representation to all translates of $D_{-}$. A simple integration by parts shows for any $f$ in $D_{A}$ that

$$
\frac{d}{d t} \hat{f}_{-}(t)=i z \hat{f}_{-}(t),
$$

where $f(t)=U(t) f$. As a consequence

$$
\hat{f}_{-}(t)=e^{i z t} \hat{f}_{-} .
$$

This extends the isometry of the map to all of the translates of $D_{-}$and hence to all of $H$ since the translates of $D_{-}$are dense in $H$ (see [8]). It also follows that $U(t)$ is represented as multiplication by $e^{i z t}$ in this representation.

Step three. The map $f \rightarrow \hat{f}_{-}$is onto $L_{2}(-\infty, \infty ; N)$. In the case of the free space representation of $U_{0}(t)$ it is known that the translates of $D_{-}$fill out $L_{2}(-\infty, \infty ; N)$ in the representation space. Since $D_{-}$and translation are represented by the same objects in both the free space and incoming spectral representations, it follows that the map $f \rightarrow \hat{f}_{-}$is onto.

THeOREM 14. The scattering operator is given by

$$
\begin{aligned}
\hat{f}_{+}(z, \omega) & =\left[\delta(z) \hat{f}_{-}(z, \cdot)\right](\omega) \\
& =\hat{f}_{-}(z, \omega)-2(2 \pi)^{1 / 2} i z \int_{|\theta|=1} s(-\theta, \omega, z)^{*} \hat{f}_{-}(z, \theta) d \theta,
\end{aligned}
$$

where

$$
\psi_{-}(r \xi, z, \omega) \sim r^{-1} e^{i z r} s(\xi, \omega, z) \text { as } r \rightarrow \infty .
$$

SKETCH OF PROOF. It suffices to determine the behavior of $S(z)$ on $D_{-}$since $S$ commutes with $U(t)$ and since translates of $D_{-}$are dense 
in $H$. Now for $f$ in $D_{-}, \hat{f}_{-}=\hat{f}_{0}$ so that $s(z) \hat{f}_{-}=\hat{f}_{+}$simply becomes

$$
s(z) \hat{f}_{-}=\hat{f}_{-}+\left(f, \psi_{-}\right), \quad f \in D_{-} .
$$

A straightforward calculation now yields (3.12). This is roughly what one expects from the classical theory and shows in particular that $\delta(z)$ differs from the identity on $N$ by an operator with a smooth kernel.

Much of the foregoing can be generalized to solutions of symmetric hyperbolic equations which satisfy conservative boundary conditions on some obstacle, provided that these boundary conditions are elliptic for the spatial part of the operator. That there are conservative boundary conditions which are not elliptic is somewhat surprising.

\section{REFERENCES}

1. A. Beurling, On two problems concerning linear transformations in Hilbert space, Acta Math. 81 (1949), 239-255. 112.

2. P. R. Halmos, Shifts on Hilbert spaces, J. Reine Angew. Math. 108 (1961), 102-

3. E. Hille and R. Phillips, Functional analysis and semi-groups, Amer. Math. Soc. Colloq. Publ. Vol. 31, Amer. Math. Soc., Providence, R. I., 1957.

4. H. Helson and D. Lowdenslager, Prediction theory and Fourier series in several variables, Acta. Math. 99 (1958), 165-202.

5. P. D. Lax, Remark on the preceding paper of Koosis, Comm. Pure Appl. Math. 10 (1957), 617-622.

6. - Translation invariant spaces, Acta Math. 101 (1959), 163-178.

7. - Proceedings of the International Symposium on Linear Spaces, Hebrew University, Jerusalem, Pergamon Press, 1961, pp. 299-306.

8. P. D. Lax and R. S. Phillips, The wave equation in exterior domains, Bull. Amer. Math. Soc. 68 (1962), 47-49.

9. P. D. Lax, C. S. Morawetz and R. S. Phillips, The exponential decay of solutions of the wave equation in the exterior of a star-shaped obstacle, Bull. Amer. Math. Soc. 68 (1962), 593-595.

10. J. W. Moeller, On the spectra of some translation invariant spaces, J. Math. Anal. Appl. 4 (1962), 276-296.

11. R. S. Phillips, Spectral theory for semigroups of linear operators, Trans. Amer. Math. Soc. 71 (1951), 393-415.

12. A. Ya. Povzner and I. V. Suharevskir, Discontinuities of the Green's function of mixed problems for the wave equation, Mat. Sb. (N.S.) 51 (1960), (93) 3-26.

13. Y. Fourès and I. E. Segal, Causality and analyticity, Trans. Amer. Math. Soc. 78 (1955), 385-405.

NEW YORK UNIVERSITY AND

STANFORD UNIVERSITY 